\title{
Irinotecan monotherapy as third-line treatment for advanced gastric cancer refractory to fluoropyrimidines, platinum, and taxanes
}

\author{
Takashi Nishimura $^{1,2} \cdot$ Satoru Iwasa $^{1} \cdot$ Kengo Nagashima $^{3} \cdot$ Natsuko Okita $^{1} \cdot$ \\ Atsuo Takashima ${ }^{1} \cdot$ Yoshitaka Honma $^{1} \cdot$ Ken Kato $^{1} \cdot$ Tetsuya Hamaguchi $^{1}$.

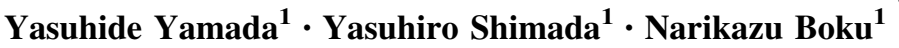

Received: 2 September 2016/Accepted: 8 November 2016/Published online: 17 November 2016

(c) The International Gastric Cancer Association and The Japanese Gastric Cancer Association 2016

\begin{abstract}
Background Because standard chemotherapy for advanced gastric cancer consists of oral fluoropyrimidines plus platinum as first-line therapy, with paclitaxel plus ramucirumab as the second line, irinotecan is usually positioned as third-line chemotherapy in clinical practice in Japan. Methods A retrospective evaluation was conducted to determine the efficacy and safety of irinotecan as third-line chemotherapy for advanced gastric cancer in patients refractory or intolerant to fluoropyrimidines, platinum, and taxanes.

Results Between February 2008 and December 2013, 52 patients received third-line irinotecan monotherapy. Among the 32 patients with measurable lesions, 1 patient achieved a confirmed partial response and 6 patients had stable disease. The overall response rate was $3 \%$ and the disease control rate was $22 \%$. Median progression-free survival was 2.3 months [95\% confidence interval (CI), 1.8-2.8] and median overall survival was 4.0 months (95\% CI, 2.6-5.3). The most common adverse events of grade 3 severity or higher were neutropenia $(27 \%)$, febrile neutropenia (12\%), anorexia (12\%), and diarrhea (6\%). Although no treatment-related deaths occurred, 2 patients
\end{abstract}

Satoru Iwasa

siwasa@ncc.go.jp

1 Gastrointestinal Medical Oncology Division, National Cancer Center Hospital, 5-1-1 Tsukiji, Chuo-ku, Tokyo 104-0045, Japan

2 Department of Gastroenterology and Hepatology, The Jikei University School of Medicine, Tokyo, Japan

3 Department of Global Clinical Research, Graduate School of Medicine, Chiba University, Chiba, Japan
(4\%) died of disease progression within 30 days after the last administration of irinotecan.

Conclusion Irinotecan monotherapy appears to be tolerated but was shown to have modest activity as third-line chemotherapy for advanced gastric cancer.

Keywords Irinotecan · Third-line chemotherapy · Gastric cancer

\section{Introduction}

Gastric cancer is a major cause of cancer-related deaths worldwide, and it remains the second leading cause of cancer-related deaths in Japan [1]. Although the mortality rate has been declining through early detection and curative surgery, patients are often diagnosed at an unresectable stage of disease. Furthermore, between $40 \%$ and $60 \%$ of patients experience a relapse, even after curative resection $[2,3]$.

Standard palliative treatment for patients with advanced gastric cancer is chemotherapy, which improves survival and quality of life compared with best supportive care alone. Many randomized controlled trials have demonstrated the survival benefits of first-line treatment consisting of a fluoropyrimidine plus platinum with or without epirubicin or docetaxel, which is the standard first-line chemotherapy for advanced gastric cancer worldwide [4-9].

Recent phase III trials showed that second-line chemotherapy with irinotecan or docetaxel produced significant and clinically meaningful improvements in overall survival (OS), relative to best supportive care in patients with advanced gastric cancer [10,11]. Weekly paclitaxel is also considered to be an option for second-line 
chemotherapy, with response rates (RR) of $13-20 \%$, median OS of 5-9 months, and modest toxicity [12-16]. In the WJOG4007 trial that compared paclitaxel with irinotecan as second-line treatment for patients with advanced gastric cancer refractory to fluoropyrimidines plus platinum, OS did not differ between the two treatment arms [15]. However, from the perspective of toxicity, paclitaxel is generally preferred to irinotecan as secondline treatment. Weekly paclitaxel is widely used in clinical practice in Japan as well as in the control arm of global clinical trials of second-line chemotherapy. Furthermore, in the RAINBOW trial, which compared ramucirumab plus paclitaxel with placebo plus paclitaxel as second-line treatment for advanced gastric cancer refractory to fluoropyrimidines plus platinum (with or without an anthracycline), the combination of ramucirumab with paclitaxel significantly improved OS compared with placebo plus paclitaxel. This regimen has been adopted as the new standard second-line treatment for patients with advanced gastric cancer [16].

The median OS of patients treated with weekly paclitaxel and irinotecan in the WJOG4007 trial was more than 9 months, which is longer than that of other phase III trials outside Japan. Because $75 \%$ and $60 \%$ of patients in the paclitaxel and irinotecan arms, respectively, received postprotocol cross-over chemotherapy, the prolonged OS in this trial was primarily attributed to the high proportion of patients who underwent subsequent chemotherapy. Similarly, in the RAINBOW trial, irinotecan was commonly used as third-line chemotherapy. In the RAINBOW trial, Asian patients received third-line chemotherapy more frequently and had longer OS compared with non-Asian patients. Moreover, each of four types of chemotherapyfluoropyrimidines, platinum, taxanes, and irinotecanwere independently associated with longer OS in patients with advanced gastric cancer [17]. Thus, the use of irinotecan as third-line chemotherapy has become common practice in Japan after the failure of fluoropyrimidines, platinum, and taxanes. However, there have been few reports on the efficacy and toxicity of irinotecan in a thirdline setting.

The aim of this study was to evaluate the efficacy and safety of irinotecan as third-line chemotherapy in advanced gastric cancer patients refractory or intolerant to fluoropyrimidines plus platinum and second-line taxanes.

\section{Patients and methods}

\section{Patients}

This retrospective study enrolled 52 patients with advanced gastric cancer who received irinotecan as third-line chemotherapy between February 2008 and December 2013 at the National Cancer Center Hospital, Tokyo. Selection criteria were as follows: histologically confirmed gastric adenocarcinoma with distant metastases or recurrent disease; failure of first-line chemotherapy with an oral fluoropyrimidine ( $\mathrm{S}-1$ or capecitabine) plus platinum (cisplatin or oxaliplatin), and second-line chemotherapy with a taxane (paclitaxel or docetaxel); no prior use of irinotecan; an Eastern Cooperative Oncology Group (ECOG) performance status (PS) of $0-2$; adequate organ function; no other type of cancer; and no serious clinical complications. This retrospective study was conducted under an approval by the institutional review board according to the Japanese ethical guidelines for epidemiological research.

\section{Treatment methods}

Irinotecan $\left(150 \mathrm{mg} / \mathrm{m}^{2}\right)$ was administered as a 90 -min intravenous infusion every 2 weeks until disease progression, unacceptable toxicity, or the patient's refusal occurred. Dose reductions of irinotecan, including the initial dose, and treatment delays were decided at each physician's discretion according to the patient's general condition, organ function, and the severity of hematological and nonhematological toxicities. In general, dose reduction and treatment delays were implemented in the case of grade 4 neutropenia, grade 3 or higher febrile neutropenia, and unacceptable grade 2 or higher diarrhea, nausea, or anorexia.

\section{Evaluation and statistical analysis}

Tumor responses were evaluated according to Response Evaluation Criteria in Solid Tumors (RECIST), version 1.1 [18]. Toxicity was assessed using the Common Terminology Criteria for Adverse Events (CTCAE), version 4.0. Progression-free survival (PFS) was defined as the time from the first day of treatment until the time of disease progression or death from any cause. PFS was censored at the date of the last visit for those patients who were alive without documented disease progression. OS was defined as time from the initiation of treatment to the date of death or censor at the last follow-up visit. For patient characteristics, disease response, and adverse events, summary statistics were constructed using frequencies and proportions for categorical data and medians and ranges for continuous variables. Survival curves were drawn by the Kaplan-Meier method. Confidence intervals (CI) for median PFS and OS were calculated with the Greenwood formula [19]. The Cox regression model was used to assess the prognostic factors for OS. $P$ values $<0.05$ by the twotailed test were considered to denote significance. IBM SPSS Statistics version 20.0 (SPSS, Chicago, IL, USA) was used for all statistical analyses. 


\section{Results}

\section{Patient characteristics}

Patient characteristics are shown in Table 1. The median age was 65 years (range, 41-78 years), and the majority of patients were male $(79 \%)$. About half the patients were histologically diagnosed with diffuse-type adenocarcinoma (48\%). First-line chemotherapy was S-1 plus cisplatin for 44 patients (85\%), S-1 plus oxaliplatin for $5(10 \%)$, and capecitabine plus cisplatin for $3(6 \%)$. Second-line chemotherapy was paclitaxel for 50 patients (96\%) and docetaxel for $2(4 \%)$. Overall RRs and disease control rates (DCR) were $43 \%$ and $80 \%$, respectively, in first-line chemotherapy with a fluoropyrimidine plus platinum, and $9 \%$ and 59\%, respectively, in second-line chemotherapy with a taxane. The median PFS was 7.6 months (95\% CI, 5.7-9.5) during first-line chemotherapy and 3.2 months $(95 \%$ CI, 2.2-4.2) during second-line chemotherapy. Ten patients (19\%) had an ECOG PS of 2. Metastatic sites were lymph nodes (69\%), peritoneum (58\%), and liver (46\%); 32 patients (62\%) had measurable lesions, whereas the other 20 patients had only nonmeasurable lesions such as peritoneal dissemination and bone metastases. The median serum albumin level was $3.5 \mathrm{mg} / \mathrm{dl}$ (range, 2.4-4.3), and the median duration from first- to third-line chemotherapy was 12.9 months (range, 3.4-34.6).

\section{Treatment exposure}

Irinotecan was administered for a median of 3 doses (range, 1-25). The initial dose of irinotecan was reduced for 25 (48\%) patients for reasons including moderate peritoneal carcinomatosis without ileus and/or massive ascites in 14 patients, ECOG PS of 2 in 4 patients, and other reasons such as advanced age in 7 patients. Among 27 patients who received the planned initial dose of irinotecan, dose reductions were required in 7 patients during the treatment course. Reasons for the dose reduction in these 7 patients were grade 4 neutropenia $(n=1)$, grade 3 neutropenia $(n=1)$, grade 3 diarrhea $(n=1)$, grade 2 diarrhea $(n=1)$, grade 3 anorexia $(n=1)$, grade 2 anorexia $(n=3)$, and grade 3 febrile neutropenia $(n=2)$. The median relative dose intensity of irinotecan for all patients was $58 \mathrm{mg} / \mathrm{m}^{2} /$ week, corresponding to $77 \%$ of the planned initial dose of $75 \mathrm{mg} / \mathrm{m}^{2} /$ week.

\section{Reasons for treatment discontinuation}

Treatment discontinuation resulted from disease progression in 47 patients (90\%), unacceptable toxicity in 4 patients, and refusal of further treatment by 1 patient. Among the 4 patients with unacceptable toxicity, 2 had grade 3 anorexia,
Table 1 Patient characteristics

\begin{tabular}{|c|c|c|}
\hline Characteristics & $n$ & $\%$ \\
\hline Age (years), median (range) & $65(41-78)$ & \\
\hline \multicolumn{3}{|l|}{ Gender } \\
\hline Male & 41 & 79 \\
\hline Female & 11 & 21 \\
\hline \multicolumn{3}{|l|}{ PS } \\
\hline 0 & 2 & 4 \\
\hline 1 & 40 & 77 \\
\hline 2 & 10 & 19 \\
\hline \multicolumn{3}{|l|}{ Prior gastrectomy } \\
\hline Yes & 18 & 35 \\
\hline No & 34 & 65 \\
\hline \multicolumn{3}{|l|}{ Histology } \\
\hline Intestinal type & 23 & 44 \\
\hline Diffuse type & 25 & 48 \\
\hline Unknown & 4 & 8 \\
\hline \multicolumn{3}{|l|}{ HER2 status } \\
\hline Positive & 2 & 4 \\
\hline Negative & 22 & 42 \\
\hline Unknown & 25 & 48 \\
\hline \multicolumn{3}{|l|}{ UGT1A1 $(* 6, * 28)$} \\
\hline Homozygous/double heterozygous & 2 & 4 \\
\hline Wild type/single heterozygous & 31 & 60 \\
\hline Unknown & 19 & 37 \\
\hline \multicolumn{3}{|l|}{ Number of metastatic sites } \\
\hline 1 & 14 & 27 \\
\hline 2 & 25 & 48 \\
\hline$\geq 3$ & 13 & 25 \\
\hline \multicolumn{3}{|l|}{ Site of metastasis } \\
\hline Liver & 24 & 46 \\
\hline Lung & 5 & 10 \\
\hline Lymph node & 36 & 69 \\
\hline Peritoneum & 30 & 58 \\
\hline \multicolumn{3}{|l|}{ Target lesion } \\
\hline Yes & 32 & 62 \\
\hline No & 20 & 39 \\
\hline \multicolumn{3}{|l|}{ First-line chemotherapy } \\
\hline S-1 plus cisplatin & 44 & 85 \\
\hline Capecitabine plus cisplatin & 3 & 6 \\
\hline S-1 plus oxaliplatin & 5 & 10 \\
\hline \multicolumn{3}{|l|}{ Second-line chemotherapy } \\
\hline Paclitaxel & 50 & 96 \\
\hline Docetaxel & 2 & 4 \\
\hline Serum albumin (mg/dl), median (range) & $3.5(2.4-4.3)$ & \\
\hline $\begin{array}{l}\text { Duration from first- to third-line } \\
\text { chemotherapy (months), median (range) }\end{array}$ & $12.9(3.4-34.6)$ & \\
\hline
\end{tabular}


1 had febrile neutropenia and grade 2 diarrhea, and 1 had grade 3 peripheral neuropathy. After discontinuing irinotecan, 11 patients $(21 \%)$ received the subsequent chemotherapies: taxanes in 5 patients, a fluoropyrimidine plus platinum in 3 patients, a fluoropyrimidine alone in 2 patients, and a fluoropyrimidine plus paclitaxel in 1 patient.

\section{Response and survival}

Responses were evaluated in 32 patients $(62 \%)$ who had measurable lesions. One patient achieved a partial response and 6 patients showed stable disease, resulting in a RR of 3\% and a DCR of $22 \%$ (Table 2). After a median follow-up period of 4.8 months for survivors (range, 0.3-21.1 months), median PFS was 2.3 months (95\% CI, 1.8-2.8), and median OS was 4.0 months (95\% CI, 2.5-5.5) (Fig. 1).

\section{Toxicity}

The hematological and nonhematological adverse events associated with irinotecan treatment are listed in Table 3. The most common grade 3 or higher hematological toxicity was neutropenia (27\%), followed by anemia (19\%). Grade 3 or

Table 2 Response of patients with measurable lesion $(n=32)$

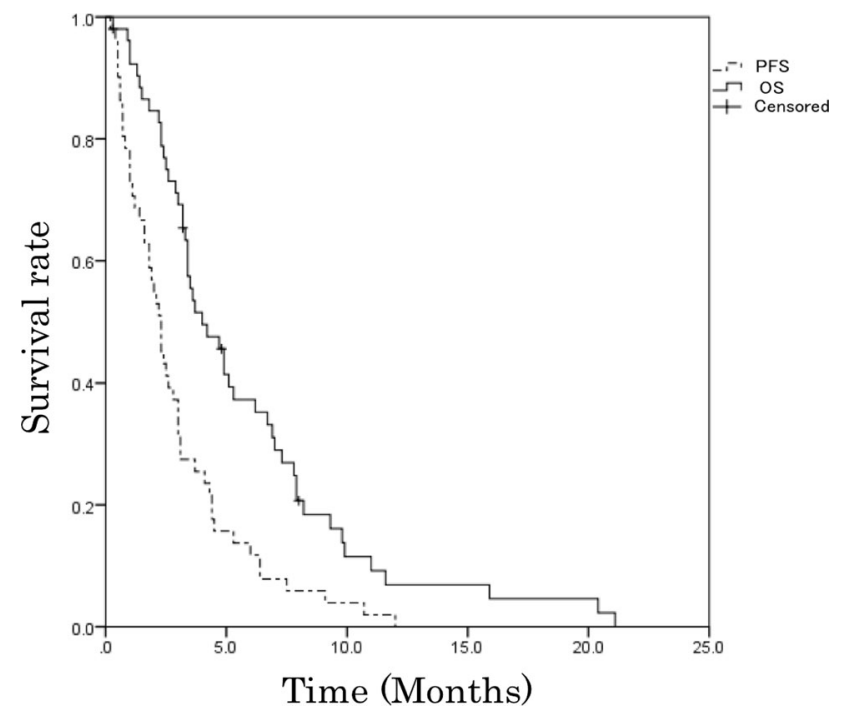

Fig. 1 Kaplan-Meier plot of progression-free survival $(P F S)$ and overall survival $(O S)$ higher nonhematological toxicities included anorexia (12\%), diarrhea (6\%), and nausea (4\%). Febrile neutropenia developed in 6 patients (12\%). Two patients (4\%) died of disease progression within 30 days of the last administration of irinotecan. There were no treatment-related deaths.

\section{Relationship between UGT1A1 gene polymorphism and adverse events}

Among 52 patients, 33 patients were tested for uridine diphosphate-glucuronosyltransferase (UGT) 1A1 gene polymorphism as a risk factor for severe adverse events. We classified the genotype of UGTIAl into three types according to the combination of $U G T 1 A I^{*} 6$ and UGT1A1 $1 * 28$ genes: wild-type group $(* 1 / * 1)$, heterozygous group $(* 28 / * 1$ and $* 6 / * 1)$, and homozygous/double heterozygous group $(* 28 / * 28, * 6 / * 6$, and $* 28 / * 6)$. The frequencies of those genotypes were $52 \%(n=17), 42 \%$ $(n=14)$, and $6 \%(n=2)$, respectively. Grade 3 or 4 neutropenia occurred in $12 \%$ (2/17), $21 \%$ (3/14), and $100 \%$ $(2 / 2)$ of patients in each group, and febrile neutropenia developed in $6 \%(1 / 17), 21 \%(3 / 14)$, and $100 \%(2 / 2)$, respectively (Table 4$)$.

\section{Univariate analysis for independent prognostic factors}

Univariate analyses for prognostic factors of OS are shown in Table 5. Risk factors for low OS were ECOG PS 2 $(P=0.003)$, serum albumin level less than $3.5 \mathrm{~g} / \mathrm{dl}$ $(P<0.001)$, three or more metastatic sites $(P=0.001)$, and a duration of less than 12.9 months between the start of first-line and the start of third-line chemotherapy $(P=0.003)$.

\section{Discussion}

Despite the lack of evidence showing a survival benefit of third-line chemotherapy for advanced gastric cancer compared with best supportive care, third-line chemotherapy has been used in 15-30\% of patients in Korean studies and in $72-90 \%$ of patients in Japanese phase III trials [15, 20-22]. The addition of salvage chemotherapy to best supportive care improved OS $(\mathrm{HR}=0.81 ; 95 \% \mathrm{CI}$, $0.450-1.464)$ in advanced gastric cancer patients who had received two or more prior chemotherapies [11].

Other retrospective analyses of third-line chemotherapy in advanced gastric cancer have reported median PFS of 1.9-3.5 months, median OS of 3.6-6.9 months, an overall RR of $5.9-23.2 \%$, and a DCR of 39.1-65.9\% [21-26]. Shimoyama et al. [23] evaluated third-line weekly paclitaxel in 85 patients with advanced gastric cancer who were 
Table 3 Adverse event

\begin{tabular}{|c|c|c|c|c|c|c|}
\hline & \multicolumn{4}{|c|}{ Grade } & \multirow{2}{*}{$\begin{array}{l}\text { All grades } \\
(\%)\end{array}$} & \multirow{2}{*}{$\begin{array}{l}\text { Grade } \\
3 / 4(\%)\end{array}$} \\
\hline & 1 & 2 & 3 & 4 & & \\
\hline \multicolumn{7}{|l|}{ Hematological } \\
\hline Leukopenia & 12 & 10 & 8 & 4 & 62 & 23 \\
\hline Neutropenia & 1 & 9 & 10 & 4 & 46 & 27 \\
\hline Anemia & 18 & 23 & 10 & 0 & 98 & 19 \\
\hline Thrombocytopenia & 0 & 0 & 0 & 0 & 0 & 0 \\
\hline \multicolumn{7}{|l|}{ Nonhematological } \\
\hline Nausea & 18 & 9 & 2 & 0 & 56 & 4 \\
\hline Vomiting & 10 & 3 & 1 & 0 & 27 & 2 \\
\hline Anorexia & 21 & 18 & 6 & 0 & 87 & 12 \\
\hline Diarrhea & 11 & 9 & 3 & 0 & 44 & 6 \\
\hline Stomatitis & 4 & 0 & 0 & 0 & 8 & 0 \\
\hline Fatigue & 32 & 15 & 1 & - & 92 & 2 \\
\hline Febrile neutropenia & - & - & 6 & 0 & 12 & 12 \\
\hline Alopecia & 15 & 13 & - & - & 54 & - \\
\hline AST elevation & 14 & 3 & 0 & 0 & 33 & 0 \\
\hline ALT elevation & 6 & 1 & 0 & 0 & 14 & 0 \\
\hline Bilirubin elevation & 1 & 1 & 0 & 0 & 4 & 0 \\
\hline Hyponatremia & 20 & 1 & 4 & 0 & 48 & 8 \\
\hline
\end{tabular}

$A S T$ aspartate transaminase, $A L T$ alanine transaminase

Table 4 Relationship between UGT1A1 gene polymorphism and adverse events

\begin{tabular}{|c|c|c|c|c|c|c|}
\hline & \multicolumn{2}{|l|}{ Wild type } & \multicolumn{2}{|c|}{ Heterozygous } & \multicolumn{2}{|c|}{ Homozygous/double heterozygous } \\
\hline & \multicolumn{2}{|l|}{$(n=17)$} & \multicolumn{2}{|l|}{$(n=14)$} & \multicolumn{2}{|l|}{$(n=2)$} \\
\hline & $\begin{array}{l}\text { All grades } \\
n(\%)\end{array}$ & $\begin{array}{l}\text { Grade } 3 / 4 \\
n(\%)\end{array}$ & $\begin{array}{l}\text { All grades } \\
n(\%)\end{array}$ & $\begin{array}{l}\text { Grade } 3 / 4 \\
n(\%)\end{array}$ & $\begin{array}{l}\text { All grades } \\
n(\%)\end{array}$ & $\begin{array}{l}\text { Grade } 3 / 4 \\
n(\%)\end{array}$ \\
\hline Leukopenia & $10(59)$ & $2(12)$ & $10(71)$ & $4(29)$ & $2(100)$ & $2(100)$ \\
\hline Neutropenia & $6(25)$ & $2(12)$ & $7(50)$ & $3(21)$ & $2(100)$ & $2(100)$ \\
\hline Anemia & 17 (100) & $2(12)$ & $13(94)$ & $3(21)$ & $2(100)$ & $2(100)$ \\
\hline Thrombocytopenia & $0(0)$ & $0(0)$ & $0(0)$ & $0(0)$ & $0(0)$ & $0(0)$ \\
\hline Nausea & $5(29)$ & $0(0)$ & $12(86)$ & $1(7)$ & $1(50)$ & $0(0)$ \\
\hline Vomiting & $2(12)$ & $0(0)$ & $7(50)$ & $0(0)$ & $0(0)$ & $0(0)$ \\
\hline Anorexia & $13(76)$ & $2(12)$ & $13(94)$ & $3(21)$ & $2(100)$ & $0(0)$ \\
\hline Diarrhea & $7(41)$ & $2(12)$ & $7(50)$ & $0(0)$ & $1(50)$ & $0(0)$ \\
\hline Stomatitis & $1(6)$ & $0(0)$ & $2(14)$ & $0(0)$ & $0(0)$ & $0(0)$ \\
\hline Fatigue & $16(94)$ & $0(0)$ & $13(94)$ & $1(7)$ & $2(100)$ & $0(0)$ \\
\hline Febrile neutropenia & $1(6)$ & $1(6)$ & $3(21)$ & $3(21)$ & $2(100)$ & $2(100)$ \\
\hline
\end{tabular}

refractory to fluoropyrimidines, irinotecan, and cisplatin. The median PFS and OS were 3.5 and 6.6 months, respectively, and the overall RR was $23.2 \%$. In another study by Kang et al. using irinotecan combined with 5-fluorouracil and leucovorin as third-line therapy in 158 patients, the median PFS and OS were 2.1 and 5.6 months, respectively, and the overall RR was $9.6 \%$ [24]. The present study demonstrates that the RR and DCR were as low as $3 \%$ and $22 \%$, respectively, and median OS was 4.0 months (95\% CI, 2.5-5.5) in 52 patients who were refractory or intolerant to previous treatment with fluoropyrimidines, platinum, and taxanes. These results appear to be inferior to the RR and OS rates of previous studies [21-26]. Because impaired bowel passage and ascites caused by peritoneal metastasis are known to induce severe toxicity to irinotecan, patients with a poor condition such 
Table 5 Univariate analysis for overall survival

\begin{tabular}{|c|c|c|c|c|}
\hline \multirow[t]{2}{*}{ Covariates } & \multirow[t]{2}{*}{ MST (months) } & \multicolumn{3}{|c|}{ Univariate analysis } \\
\hline & & HR & $95 \% \mathrm{CI}$ & $P$ value \\
\hline \multicolumn{5}{|l|}{ Age (years) } \\
\hline$<65$ & 3.7 & 1 & & \\
\hline$\geq 65$ & 4.7 & 0.68 & $0.37-1.24$ & 0.20 \\
\hline \multicolumn{5}{|l|}{ Gender } \\
\hline Female & 3.2 & 1 & & \\
\hline Male & 4.9 & 0.65 & $0.33-1.29$ & 0.22 \\
\hline \multicolumn{5}{|l|}{ PS } \\
\hline $0-1$ & 4.9 & 1 & & \\
\hline$\geq 2$ & 2.1 & 2.92 & $1.42-6.00$ & 0.004 \\
\hline \multicolumn{5}{|l|}{ Histology } \\
\hline Intestinal type & 4.9 & 1 & & \\
\hline Diffuse type & 3.6 & 1.14 & $0.63-2.07$ & 0.67 \\
\hline \multicolumn{5}{|l|}{ Prior gastrectomy } \\
\hline No & 3.7 & 1 & & \\
\hline Yes & 4.7 & 0.71 & $0.38-1.31$ & 0.27 \\
\hline \multicolumn{5}{|c|}{ Number of metastatic sites } \\
\hline $1-2$ & 5.3 & 1 & & \\
\hline$\geq 3$ & 2.9 & 3.16 & $1.59-6.30$ & 0.001 \\
\hline \multicolumn{5}{|c|}{ Peritoneal metastasis } \\
\hline No & 4.8 & 1 & & \\
\hline Yes & 3.6 & 1.14 & $0.64-2.01$ & 0.67 \\
\hline \multicolumn{5}{|l|}{ Target lesion } \\
\hline No & 4.9 & 1 & & \\
\hline Yes & 3.9 & 1.29 & $0.71-2.34$ & 0.40 \\
\hline \multicolumn{5}{|l|}{ Serum albumin } \\
\hline$<3.5$ & 2.5 & 1 & & \\
\hline$\geq 3.5$ & 6.9 & 0.33 & $0.18-0.60$ & $<0.001$ \\
\hline \multicolumn{5}{|c|}{ Time from first-line to third-line chemotherapy } \\
\hline$\geq 12.9$ months & 6.7 & 1 & & \\
\hline$<12.9$ months & 2.9 & 2.90 & $1.56-5.38$ & 0.001 \\
\hline
\end{tabular}

$M S T$ median survival time, $H R$ hazard ratio, $P S$ performance status

as peritoneal metastasis were included in this study and required an initial dose reduction. Nevertheless, irinotecan monotherapy as the second-line chemotherapy produced an RR of $13.6 \%$ and median PFS of 2.3 months in the WJOG4007 trial. Therefore, PFS was similar in the present study to that in the WJOG4007 trial.

Shim et al. reported on the prognostic factors affecting poor survival following third-line chemotherapy. Poor PS (ECOG PS $\geq 2$ ), low serum albumin $(<4.0 \mathrm{~g} / \mathrm{dl})$, poor histological grade, and a shorter duration of second-line chemotherapy ( $<2.7$ months) were factors related to poor survival outcomes [22]. Another clinical trial demonstrated that poor PS (ECOG PS $\geq 2$ ), an increased number of metastatic organs $(\geq 3)$, and a short time duration between first-line and third-line chemotherapy (10.9 months) were independent factors for poor prognosis in multivariate analysis [11]. In this study, ECOG PS 0-1, the number of metastatic sites (1-2), serum albumin level $(3.5 \mathrm{~g} / \mathrm{dl}$ or more), and duration from first-line to third-line chemotherapy (at least 12.9 months) were associated with prolonged OS in univariate analysis. These findings suggest that a good PS is the most important prognostic factor for third-line chemotherapy, as demonstrated for secondline treatment $[20,27-30]$. By restricting analysis to patients who fulfilled the following clinical conditions (PS $0-1,<3$ metastatic sites, serum albumin $\geq 3.5 \mathrm{~g} / \mathrm{dl}$, and $\geq 12.9$ months between first- and third-line chemotherapy, the median PFS and OS were 3.1 and 9.1 months, respectively. In contrast, patients with at least one risk factor had a median PFS and OS of 2.0 and 3.4 months, respectively. This finding suggests that patients should be carefully selected for third-line chemotherapy for advanced gastric cancer.

In the present study, the most frequent grade 3-4 hematological toxicities caused by irinotecan were neutropenia (27\%) and anemia (19\%), with 12\% developing febrile neutropenia. The most common grade 3-4 nonhematological toxicities were anorexia $(12 \%)$, diarrhea $(6 \%)$, and nausea (4\%). Although patients had been previously heavily treated with two regimens, these incidences of grade 3-4 toxicities were not very different from those in previous studies of irinotecan as second-line treatment $[10,11,15]$, thus highlighting the feasibility of irinotecan as third-line treatment. However, because many of patients in this study received a reduced dose of irinotecan, it is suggested that the appropriate dose of irinotecan should be based on the patient's condition. Furthermore, we also evaluated the adverse events according to UGTIAl gene polymorphism. UGT1Al has an important function in the metabolism of irinotecan. Several studies suggested that $U G T 1 A 1 * 6$ and $* 28$ genotypes were associated with a increase in the occurrence of adverse events such as neutropenia in irinotecan chemotherapy [31-33]. Although in this study there were only two patients in the homozygous/double-heterozygous group, which is a risk factor for severe adverse events, both these patients had grade 3-4 neutropenia and febrile neutropenia. These results might imply that administration of irinotecan as third-line treatment for advanced gastric cancer patients with homozygous or double-heterozygous type should be withdrawn in terms of safety and efficacy.

There are several limitations to this study. First, it was performed retrospectively, with the initial dose of irinotecan determined by each physician's judgment. About half of all patients (48\%) initially received reduced doses of irinotecan from the first cycle, and $27 \%$ of patients received only one cycle of irinotecan as a third-line treatment, possibly because of the inclusion of patients in poor 
general condition such as peritoneal metastasis. Second, toxicity may have been underestimated because of the inherent characteristics of the retrospective design. Third, a small sample size from a single-center study is an obvious limitation.

In conclusion, irinotecan appeared to be feasible, but only showed modest efficacy for advanced gastric cancer refractory to fluoropyrimidines, platinum, and taxanes. Therefore, irinotecan monotherapy as the third-line treatment should be carefully indicated in patients with a good PS of 0 or 1.

\section{Compliance with ethical standards}

Conflict of interest Satoru Iwasa and Ken Kato have received research grants from Daiichi-Sankyo; Yasuhide Yamada has received an honorarium from Yakult; Yasuhiro Shimada received an honorarium from Daiichi-Sankyo; and Narikazu Boku had an honorarium from Yakult and Daiichi-Sankyo during the conduct of the study.

Human rights statement and informed consent All procedures followed were in accordance with the ethical standards of the responsible committee on human experimentation (institutional and national) and with the Helsinki Declaration of 1964 and later versions. Informed consent or a substitute for it was obtained from all patients for being included in the study.

\section{References}

1. Vital Statistics Japan (Ministry of Health, Labour and Welfare), http://ganjoho.jp/public/statistics/pub/statistics01.html. Accessed 5 May 2015.

2. D'Angelica M, Gonen M, Brennan MF, Turnbull AD, Bains M, Karpeh MS. Patterns of initial recurrence in completely resected gastric adenocarcinoma. Ann Surg. 2004;240:808-16.

3. Kim S, Lim DH, Lee J, Kang WK, MacDonald JS, Park CH, et al. An observational study suggesting clinical benefit for adjuvant postoperative chemoradiation in a population of over 500 cases after gastric resection with D2 nodal dissection for adenocarcinoma of the stomach. Int J Radiat Oncol Biol Phys. 2005;63:1279-85.

4. Cunningham D, Starling N, Rao S, Iveson T, Nicolson M, Coxon $\mathrm{F}$, et al. Capecitabine and oxaliplatin for advanced esophagogastric cancer. N Engl J Med. 2008;358:36-46.

5. Bang YJ, Van Cutsem E, Feyereislova A, Chung HC, Shen L, Sawaki A, et al. Trastuzumab in combination with chemotherapy versus chemotherapy alone for treatment of HER2-positive advanced gastric or gastro-oesophageal junction cancer (ToGA): a phase 3, open-label, randomised controlled trial. Lancet. 2010;376:687-97.

6. Kang YK, Kang WK, Shin DB, Chen J, Xiong J, Wang J, et al. Capecitabine/cisplatin versus 5-fluorouracil/cisplatin as first-line therapy in patients with advanced gastric cancer: a randomised phase III noninferiority trial. Ann Oncol. 2009;20:666-73.

7. Ajani JA, Rodriguez W, Bodoky G, Moiseyenko V, Lichinitser M, Gorbunova V, et al. Multicenter phase III comparison of cisplatin/S-1 with cisplatin/infusional fluorouracil in advanced gastric or gastroesophageal adenocarcinoma study: the FLAGS trial. J Clin Oncol. 2010;28:1547-53.

8. Ohtsu A, Shah MA, Van Cutsem E, Rha SY, Sawaki A, Park SR, et al. Bevacizumab in combination with chemotherapy as first- line therapy in advanced gastric cancer: a randomized, doubleblind, placebo-controlled phase III study. J Clin Oncol. 2011;29:3968-76.

9. Koizumi W, Narahara H, Hara T, Takagane A, Akiya T, Takagi $\mathrm{M}$, et al. S-1 plus cisplatin versus S-1 alone for first-line treatment of advanced gastric cancer (SPIRITS trial): a phase III trial. Lancet Oncol. 2008;9:215-21.

10. Thuss-Patience PC, Kretzschmar A, Bichev D, Deist T, Hinke A, Breithaupt K, et al. Survival advantage for irinotecan versus best supportive care as second-line chemotherapy in gastric cancer: a randomised phase III study of the Arbeitsgemeinschaft Internistische Onkologie (AIO). Eur J Cancer. 2011;47:2306-14.

11. Kang JH, Lee SI, Lim DH, Park KW, Oh SY, Kwon HC, et al. Salvage chemotherapy for pretreated gastric cancer: a randomized phase III trial comparing chemotherapy plus best supportive care with best supportive care alone. J Clin Oncol. 2012;30:1513-8.

12. Kodera Y, Ito S, Mochizuki Y, Fujitake S, Koshikawa K, Kanyama Y, et al. A phase II study of weekly paclitaxel as second-line chemotherapy for advanced gastric cancer (CCOG0302 Study). Anticancer Res. 2007;27:2667-71.

13. Hironaka S, Zenda S, Boku N, Fukutomi A, Yoshino T, Onozawa Y. Weekly paclitaxel as second-line chemotherapy for advanced or recurrent gastric cancer. Gastric Cancer. 2006;9:14-8.

14. Matsuda G, Kunisaki C, Makino H, Fukahori M, Kimura J, Sato $\mathrm{T}$, et al. Phase II study of weekly paclitaxel as a second-line treatment for S-1-refractory advanced gastric cancer. Anticancer Res. 2009;29:2863-7.

15. Hironaka S, Ueda S, Yasui H, Nishina T, Tsuda M, Tsumura T, et al. Randomized, open-label, phase III study comparing irinotecan with paclitaxel in patients with advanced gastric cancer without severe peritoneal metastasis after failure of prior combination chemotherapy using fluoropyrimidine plus platinum: WJOG 4007 trial. J Clin Oncol. 2013;31:4438-44.

16. Wilke H, Muro K, Van Cutsem E, Oh SC, Bodoky G, Shimada Y, et al. Ramucirumab plus paclitaxel versus placebo plus paclitaxel in patients with previously treated advanced gastric or gastrooesophageal junction adenocarcinoma (RAINBOW): a doubleblind, randomised phase 3 trial. Lancet Oncol. 2014;15:1224-35.

17. Shitara K, Matsuo K, Mizota A, Kondo C, Nomura M, Takahari $\mathrm{D}$, et al. Association of fluoropyrimidines, platinum agents, taxanes, and irinotecan in any line of chemotherapy with survival in patients with advanced gastric cancer. Gastric Cancer. 2011;14:155-60.

18. Eisenhauer EA, Therasse P, Bogaerts J, Schwartz LH, Sargent D, Ford R, et al. New response evaluation criteria in solid tumours: revised RECIST guideline (version 1.1). Eur J Cancer. 2009;45:228-47.

19. Greenwood M. The natural duration of cancer. Reports on Public Health and Medical Subjects. Lond Majesty's Station Off. 1926;33:1-26.

20. Ji SH, Lim DH, Yi SY, Kim HS, Jun HJ, Kim KH, et al. A retrospective analysis of second-line chemotherapy in patients with advanced gastric cancer. BMC Cancer. 2009;9:110.

21. Moon YW, Rha SY, Jeung HC, Kim C, Hong MH, Chang H, et al. Outcomes of multiple salvage chemotherapy for advanced gastric cancer: implications for clinical practice and trial design. Cancer Chemother Pharmacol. 2010;66:797-805.

22. Shim HJ, Yun JY, Hwang JE, Bae WK, Cho SH, Chung IJ. Prognostic factor analysis of third-line chemotherapy in patients with advanced gastric cancer. Gastric Cancer. 2011;14:249-56.

23. Shimoyama R, Yasui H, Boku N, Onozawa Y, Hironaka S, Fukutomi A, et al. Weekly paclitaxel for heavily treated advanced or recurrent gastric cancer refractory to fluorouracil, irinotecan, and cisplatin. Gastric Cancer. 2009;12:206-11. 
24. Kang EJ, Im SA, Oh DY, Han SW, Kim JS, Choi IS, et al. Irinotecan combined with 5-fluorouracil and leucovorin third-line chemotherapy after failure of fluoropyrimidine, platinum, and taxane in gastric cancer: treatment outcomes and a prognostic model to predict survival. Gastric Cancer. 2013;16:581-9.

25. Lee MJ, Hwang IG, Jang JS, Choi JH, Park BB, Chang MH, et al. Outcomes of third-line docetaxel-based chemotherapy in advanced gastric cancer who failed previous oxaliplatin-based and irinotecan-based chemotherapies. Cancer Res Treat. 2012;44:235-41.

26. Godai TI, Oshima T, Numata M, Fukahori M, Sato T, Makino H, et al. Clinical efficacy and safety of CPT-11 + CDDP therapy as third-line chemotherapy for advanced and recurrent gastric cancer. Gan To Kagaku Ryoho. 2011;38:945-9.

27. Lee J, Lim T, Uhm JE, Park KW, Park SH, Lee SC, et al. Prognostic model to predict survival following first-line chemotherapy in patients with metastatic gastric adenocarcinoma. Ann Oncol. 2007; 18:886-91.

28. Kanagavel D, Pokataev IA, Fedyanin MY, Tryakin AA, Bazin IS, Narimanov MN, et al. A prognostic model in patients treated for metastatic gastric cancer with second-line chemotherapy. Ann Oncol. 2010;21:1779-85.
29. Catalano V, Graziano F, Santini D, D’Emidio S, Baldelli AM, Rossi D, et al. Second-line chemotherapy for patients with advanced gastric cancer: who may benefit? $\mathrm{Br} \mathrm{J}$ Cancer. 2008;99:1402-7.

30. Hashimoto K, Takashima A, Nagashima K, Okazaki SS, Nakajima TE, Kato K, et al. Progression-free survival in first-line chemotherapy is a prognostic factor in second-line chemotherapy in patients with advanced gastric cancer. J Cancer Res Clin Oncol. 2010;136:1059-64.

31. Ando Y, Saka H, Ando M, Sawa T, Muro K, Ueoka H, et al Polymorphisms of UDP-glucuronosyltransferase gene and irinotecan toxicity: a pharmacogenetic analysis. Cancer Res. 2000;60:6921-6.

32. Innocenti F, Undevia SD, Iyer L, Chen PX, Das S, Kocherginsky $\mathrm{M}$, et al. Genetic variants in the UDP-glucuronosyltransferase $1 \mathrm{~A} 1$ gene predict the risk of severe neutropenia of irinotecan. J Clin Oncol. 2004;22:1382-8.

33. Minami H, Sai K, Saeki M, Saito Y, Ozawa S, Suzuki K, et al. Irinotecan pharmacokinetics/pharmacodynamics and UGT1A genetic polymorphisms in Japanese: roles of UGT1A1*6 and *28. Pharmacogenet Genomics. 2007;17:497-504. 